\title{
Isolation of LMX1a Ventral Midbrain Progenitors Improves the Safety and Predictability of Human Pluripotent Stem Cell-Derived Neural Transplants in Parkinsonian Disease
}

\author{
Isabelle R. de Luzy, ${ }^{1 *}$ Jonathan C. Niclis, ${ }^{1 *}$ - Carlos W. Gantner, ${ }^{1}$ Jessica A. Kauhausen, ${ }^{1}$ Cameron P.J. Hunt, ${ }^{1,2}$ \\ Charlotte Ermine, ${ }^{1}$ Colin W. Pouton, ${ }^{2}{ }^{-}$Lachlan H. Thompson, ${ }^{1}$ and ${ }^{\circ}$ Clare L. Parish ${ }^{1}$ \\ ${ }^{1}$ Florey Institute of Neuroscience and Mental Health, Parkville, 3052 Victoria, Australia, and ${ }^{2}$ Monash Institute of Pharmaceutical Sciences, Monash \\ University, Parkville, 3052 Victoria, Australia
}

Human pluripotent stem cells (hPSCs) are a promising resource for the replacement of degenerated ventral midbrain dopaminergic (vmDA) neurons in Parkinson's disease. Despite recent advances in protocols for the in vitro generation of vmDA neurons, the asynchronous and heterogeneous nature of the differentiations results in transplants of surprisingly low vmDA neuron purity. As the field advances toward the clinic, it will be optimal, if not essential, to remove poorly specified and potentially proliferative cells from donor preparations to ensure safety and predictable efficacy. Here, we use two novel hPSC knock-in reporter lines expressing GFP under the LMX1A and PITX3 promoters, to selectively isolate vm progenitors and DA precursors, respectively. For each cell line, unsorted, GFP ${ }^{+}$, and $\mathrm{GFP}^{-}$cells were transplanted into male or female Parkinsonian rodents. Only rats receiving unsorted cells, LMX1A-eGFP ${ }^{+}$, or PITX3-eGFP $^{-}$cell grafts showed improved motor function over 6 months. Postmortem analysis revealed small grafts from PITX3$\mathrm{eGFP}^{+}$cells, suggesting that these DA precursors were not compatible with cell survival and integration. In contrast, LMX1A-eGFP ${ }^{+}$ grafts were highly enriched for vmDA neurons, and importantly excluded expansive proliferative populations and serotonergic neurons. These LMX1A-eGFP ${ }^{+}$progenitor grafts accelerated behavioral recovery and innervated developmentally appropriate forebrain targets, whereas LMX1A-eGFP ${ }^{-}$cell grafts failed to restore motor deficits, supported by increased fiber growth into nondopaminergic target nuclei. This is the first study to use an hPSC-derived reporter line to purify vm progenitors, resulting in improved safety, predictability of the graft composition, and enhanced motor function.

Key words: cell sorting; dopamine; LMX1A; Parkinson's disease; PITX3; transplantion

Significance Statement

Clinical trials have shown functional integration of transplanted fetal-derived dopamine progenitors in Parkinson's disease. Human pluripotent stem cell (hPSC)-derived midbrain progenitors are now being tested as an alternative cell source; however, despite current differentiation protocols generating $>80 \%$ correctly specified cells for implantation, resultant grafts contain a small fraction of dopamine neurons. Cell-sorting approaches, to select for correctly patterned cells before implantation, are being explored yet have been suboptimal to date. This study provides the first evidence of using $2 \mathrm{hPSC}$ reporter lines (LMX1A-GFP and PITX3-GFP) to isolate correctly specified cells for transplantation. We show LMX1A-GFP ${ }^{+}$, but not PITX3-GFP ${ }^{+}$, cell grafts are more predictable, with smaller grafts, enriched in dopamine neurons, showing appropriate integration and accelerated functional recovery in Parkinsonian rats.

\section{Introduction}

Clinical studies, using human fetal tissue enriched with dopaminergic (DA) progenitors, have provided the necessary evidence that new DA neurons transplanted into the brains of Parkinson's disease patients can functionally integrate and alleviate motor symptoms for decades (Barker et al., 2013). Poor standardization 
procedures, limited tissue availability, and ethical consideration associated using fetal tissue have resulted in a shift toward the use of human pluripotent stem cells (PSCs) to generate donor cells. Despite significant advancements in ventral mesencephalic differentiation protocols in recent years (Kriks et al., 2011; Denham et al., 2012; Kirkeby et al., 2012; Niclis et al., 2017a), cultures remain asynchronous and heterogeneous, with even the most advanced current protocols reporting that $\sim 10 \%-15 \%$ of cells fail to adopt a ventral midbrain ( vm) progenitor fate at times amenable to transplantation (Kirkeby et al., 2017; Niclis et al., 2017a). While this appears to represent a relatively high specification efficiency, upon transplantation, studies report low yields of TH-expressing DA neurons within the grafts (Kriks et al., 2011; Kirkeby et al., 2012, 2017; Doi et al., 2014; Samata et al., 2016; Niclis et al., 2017b). These observations indicate significant expansion of other vm progenitors not destined to become DA neurons as well as proliferation of incorrectly specified nondopaminergic progenitors in vivo that may also pose a risk of neural overgrowths/tumors. One also recognizes the risk of incorrectly specified neuronal populations, such as serotonergic neurons within grafts, that may induce dyskinetic behaviors (Carlsson et al., 2007; Politis et al., 2011).

A key strategy to overcome such conundrums and ensure the reproducible generation of safe and predictable cell products for clinical translation is to selectively enrich for appropriately specified vm progenitors before transplantation. While a number of rodent studies have successfully isolated vm progenitors, using reporter mice/cell lines and antibodies targeted against extracellular proteins, using both FACS as well as magnetic beadactivated cell sorting (Fukuda et al., 2006; Thompson et al., 2006; Hedlund et al., 2008; Jönsson et al., 2009; Ganat et al., 2012; Nefzger et al., 2012; Bye et al., 2015), isolation from human PSC (hPSC) cultures has been met with variable success. In part, this has been due to breadth of expression of the transgene/protein, timing of expression of the gene/protein and hence progenitor isolation occurring weeks before transplantation, and/or suboptimal specificity (or availability) of antibodies for human cells (Aguila et al., 2014; Doi et al., 2014; Samata et al., 2016; Lehnen et al., 2017).

With the field rapidly advancing to the clinic (Barker et al., 2017), there is a persistent and inherent need to identify a reliable candidate marker for the enrichment of DA progenitors from hPSC-derived vm cultures. Here we assessed the capacity to isolate vm progenitors and DA precursors based upon two cardinal genes involved in vmDA development: LMX1A, an early vm determinant (Andersson et al., 2006; Yan et al., 2011); and PITX3, a gene required for the postmitotic maturation of DA progenitors (Smidt et al., 2004). Both genes have been used to isolate vm progenitors/precursors from mouse embryonic stem cell (ESC) cultures (Hedlund et al., 2008; Nefzger et al., 2012). We demonstrate that, following FACS isolation and transplantation, $\mathrm{LMX} \mathrm{A}-\mathrm{eGFP}^{+}$progenitors, but not PITX3-eGFP ${ }^{+}$DA precursor cells, resulted in a higher density of $\mathrm{TH}^{+} \mathrm{DA}$ neurons within grafts, appropriate target innervation, and consequential improved motor function, while critically eliminating proliferative and serotonergic populations from the grafts.

\footnotetext{
Support Grant. We thank Mong Tien for expert technical assistance; and Melbourne Brain Centre flow cytometry facility for support.

The authors declare no competing financial interests.

*I.R.d.L. and J.C.N. contributed equally to this work.

Correspondence should be addressed to Clare L. Parish at clare.parish@florey.edu.au.

https://doi.org/10.1523/JNEUROSCI.1160-19.2019

Copyright $\odot 2019$ the authors
}

\section{Materials and Methods}

Human ESC culture and differentiation. Human ESC H9 reporter lines, LMX1A-eGFP and PITX3-eGFP, were cultured and differentiated under xeno-free conditions as previously described (Niclis et al., 2017a). In brief, cells were cultured on Laminin-521 (10 $\mu \mathrm{g} / \mathrm{ml}$; BioLamina) and exposed to dual SMAD inhibition (SB431542, $10 \mu \mathrm{M}, 0-5$ DIV, R\&D Systems; and LDN193189, $200 \mathrm{~nm}, 0-11$ DIV, Stemgent) to promote neuralization. Regionalization to a vm floor plate identity was achieved by exposure to Sonic hedgehog C25II (100 ng/ml; R\&D Systems) and Purmorphamine ( $2 \mu \mathrm{M}$; Stemgent) from 1-7 DIV, in addition to Wnt agonist, CHIR 99021 (3 $\mu \mathrm{M}$; Stemgent, 3-13 DIV). From 11 DIV, cells were cultured in a "maturation media" consisting of NBB27 supplemented with GDNF (20 ng/ml; R\&D Systems), BDNF (20 ng/ml, R\&D Systems), TGF $\beta 3$ ( $1 \mathrm{ng} / \mathrm{ml}$; PeproTech), DAPT (10 $\mu \mathrm{M}$, Sigma-Aldrich), ascorbic acid (200 nм; Sigma-Aldrich), and dibutyryl cAMP (0.05 mm; Tocris Bioscience).

FACS. At 21 DIV, cultures rich in vm progenitor/precursors were dissociated in Accutase (Innovative Cell Technologies) to a single-cell suspension and resuspended into a sorting buffer containing $90 \%$ maturation media, $10 \% \mathrm{KO}$ serum replacement, and ROCK inhibitor Y-27632 $(10 \mu \mathrm{M}$, Tocris Bioscience $)$ to a final density of $5-8 \times 10^{6}$ cells $/ \mathrm{ml}$. Cells were filtered through cell strainer caps $(35 \mu \mathrm{m}$ mesh, Falcon) to remove the presence of cell doublets and clusters. Sorting was performed on a MoFlo cell sorter (Beckman Coulter) or FACS Aria III using a $100 \mu \mathrm{M}$ nozzle, sheath pressure of 17-22 psi. A fraction of cells were passed through the cell sorter, without selection, forming the unsorted group, whereas $\mathrm{eGFP}^{+}$and $\mathrm{eGFP}^{-}$cells were isolated using a highly restrictive gating strategy to exclude doublets and debris based on forward and side-scatter parameters, as well as dead cells using the viability marker DAPI. Background autofluorescence was compensated for by an empty autofluorescence channel (FL2-580/30), and a H9 parental cell line at the same stage of differentiation was used as a $\mathrm{GFP}^{-}$control (data not shown). FACS-isolated cell fractions were (1) replated at 300,000 cells $/ \mathrm{cm}^{2}$ in 96-well plates, (2) passed back through the FACS machine (both targeted at confirming efficacy of the FACS isolation), or (3) resuspended in maturation media containing ROCK inhibitor (Y27632, $10 \mu \mathrm{M}$, Sigma-Aldrich) at 100,000 cells/ $\mu$ l and stored on ice until the time of transplantation. Flow cytometric analysis was conducted using FlowJo software.

6-OHDA lesioning and cell transplantation. All animal procedures were conducted in agreement with the Australian National Health and Medical Research Council's published Code of Practice for the Use of Animals in Research, and experiments were approved by the Florey Institute of Neuroscience and Mental Health Animal Ethics committee. Animals (of either sex) were group housed on a 12:12 h light/dark cycle with ad libitum access to food and water. Surgeries were performed on athymic nude rats and nude mice under $2 \%$ isoflurane anesthesia. Unilateral 6-OHDA lesions of the rat medial forebrain bundle $(3.5 \mu \mathrm{l}, 3.2 \mu \mathrm{g} / \mu \mathrm{l})$ or mouse substantia nigra pars compacta $(1.5 \mu \mathrm{l}, 1.6 \mu \mathrm{g} / \mu \mathrm{l})$ were performed to ablate the host midbrain dopamine pathways, as previously described (Kauhausen et al., 2013; Niclis et al., 2017a).

Six weeks after lesioning, animals received a unilateral injection of either hPSC-derived LMX1a-eGFP vm progenitors or PITX3-eGFP DA precursors into the denervated striatum. For each cell line, grafts were of unsorted, $\mathrm{GFP}^{+}$, or $\mathrm{GFP}^{-}$progenitors $(1 \mu \mathrm{l} ; 100,000 \mathrm{cells} / \mu \mathrm{l})$. Stereotaxic coordinates were as follows in rats: $0.5 \mathrm{~mm}$ anterior, $2.5 \mathrm{~mm}$ lateral to bregma and $4.0 \mathrm{~mm}$ below the dura surface, and in mice: $0.5 \mathrm{~mm}$ anterior, $2.0 \mathrm{~mm}$ lateral, $3.2 \mathrm{~mm}$ ventral, as previously described (Kauhausen et al., 2013; Niclis et al., 2017b). For study design overview, see Figure 1.

Behavioral testing. Rotational asymmetry, following intraperitoneal administration of D-amphetamine sulfate ( $3.5 \mathrm{mg} / \mathrm{kg}$, Tocris Bioscience), was assessed 4 weeks after 6-OHDA lesioning. Only animals capable of $>300$ rotations in $60 \mathrm{~min}$, confirmed on 2 independent tests/rat, were included in the study. Behavioral testing was repeated at 16,20, and 24 weeks after cell grafting to assess the functional integration of the transplants.

Immunocytochemistry. In vitro cultures were fixed with $4 \% \mathrm{PFA}$ at day 21 , or at $6 \mathrm{~h}$ after FACS and in vitro replating. Rats and mice were killed by 
an overdose of sodium pentobarbitone $(100 \mathrm{mg} / \mathrm{kg})$, and transcardially perfused with Tyrode's solution followed by 4\% PFA. Brains were coronally sectioned ( $40 \mu \mathrm{m} ; 12$ series) on a freezing microtome (Leica Microsystems). Immunohistochemistry was performed in accordance to previously described methods (Somaa et al., 2017). Primary antibodies and dilutions were as follows: DAPI (1:5000; Sigma-Aldrich), rabbit antiOTX2 (1:4000; Millipore), goat anti-FOXA2 (1:200; Santa Cruz Biotechnology), chicken anti-GFP (1:1000; Abcam), rabbit anti-GFP (1:20,000; Abcam), sheep anti-TH (1:800, Pelfreeze), rabbit anti-TH (1:1000; Pelfreeze), rabbit anti-BARHL1 (1:300, Novus Biologicals), sheep antiPITX2 (1:500, R\&D Systems), mouse anti-neural cell adhesion molecule (NCAM, 1:500; Santa Cruz Biotechnology), rabbit anti-NeuN (1:100; R\&D Systems), mouse anti-GAD67 (1:100, Millipore), goat antiChAT (1:100, Millipore), mouse anti-dopamine beta-hydroxylase (DBH, 1:5000, Millipore, MAB308), rabbit anti-GFAP (1:500, Dako), mouse anti-adenomatous polyposis coli, clone CC1 (APC, 1:100, Abcam), mouse anti-rat endothelial cell antigen-1 (RECA1, 1:500, Serotec), rabbit anti-Claudin V (1:200, Abcam), rabbit anti-GIRK2 (1:500; Alomone Labs), mouse anti-calbindin (1:1000; Swant Biotech), rabbit antiKI67 (1:1000; Thermo Fisher Scientific), goat anti-doublecortin (DCX, 1:1000; Santa Cruz Biotechnology), mouse anti-human nuclear antigen (HNA, 1:300; Millipore), and rabbit anti-5-HT (1:1000; Immunostar). Secondary antibodies for direct detection were used at dilution of 1:200: DyLight 488-, 549-, or 649-conjugated donkey anti-mouse, anti-chicken, anti-rabbit, anti-goat, or anti-sheep (Jackson ImmunoResearch Laboratories).

Microscopy and quantification. Fluorescence images were captured using a Carl Zeiss Axio Observer Z.1 epifluorescence or Carl Zeiss LSM 780 confocal microscope, whereas bright and dark field images were obtained using a Leica Microsystems DM6000 microscope. Grafts were delineated based upon PSA-NCAM expression and volume estimated using the Cavalieri's principle. Total number of $\mathrm{HNA}^{+}, \mathrm{GFP}^{+}, \mathrm{TH}^{+}$, serotonergic $\left(5-\mathrm{HT}^{+}\right)$, and proliferative $\left(\mathrm{KI}_{67}{ }^{+}\right)$cells were counted from images captured at $20 \times$ magnification. To quantify GIRK $2^{+}$and calbindin ${ }^{+}$ cells within the grafts, dopamine neurons were first identified based upon TH immunoreactivity from acquired confocal images.

The density of TH- and NCAM-labeled fibers was assessed at predetermined sites within known dopaminergic (dorsolateral striatum and cortex) and nondopaminergic (periventricular thalamic nucleus and fimbria of the contralateral hippocampus) target nuclei, from compressed $z$-stacked images. Images were analyzed using ImageJ software.

Experimental design and statistical analysis. All behavior testing and histological assessments were performed with the researcher blinded to the experimental group. A total of 48 female athymic nude rats (CBHrnu) and 13 nude mice (BALB/c-Foxn $1^{\nu} / \mathrm{Arc}$ ) were transplanted from two independent differentiations (into rats and subsequently mice, with $n=$ 3-8 animals per group). All data are presented as mean \pm SEM. Statistical tests used (inclusive of one-way ANOVA, two-way ANOVA, and Student's $t$ tests), as well as number of animals/group for each analysis are stated in the figure legends. Alpha levels of $p<0.05$ were considered significant with all statistical analysis performed using GraphPad Prism.

\section{Results}

\section{Isolation of vm progenitor/precursors from LMX1A-eGFP and PITX3-eGFP human ESC lines}

eGFP reporter human ESC lines for the early vm progenitor gene LMX1A and later DA precursor gene PITX3 (Fig. $1 A-C$ ) were differentiated according to the recently developed, and clinically relevant, xenogeneic-free protocol (Niclis et al., 2017a). At the time corresponding to the FACS isolation of progenitors for transplantation, 21 DIV, the efficiency of the differentiating cultures could be confirmed in both stem cell lines by the coexpression of the forebrain-midbrain marker OTX2 and basal plate marker, FOXA2 (Fig. $1 D, E, J, K$ ), accounting for $>85 \%$ of all cells. At this time, the LMX1A-eGFP reporter line revealed that a significant proportion of the cultures also coexpressed LMX1A (i.e., $\mathrm{GFP}^{+},>70 \%$ ), as previously described (Niclis et al., 2017a), with few maturing $\mathrm{TH}^{+}$cells emerging (Fig. $1 F-I$ ). In contrast,
PITX3 expression, visible by GFP immunocytochemistry in the differentiated PITX3-e ESC cultures, showed sparse expression $(<2 \%)$ that tightly overlapped with TH (Fig. $1 L-O)$. Importantly, we showed low proportions of BARHL1+ and PITX2+ immunoreactive cells, indicative of off-target, developmentally adjacent subthalamic progenitors (Kee et al., 2017), within differentiating LMX1A-GFP (Fig. 1P) and PITX3-GFP (data not shown) cell lines.

FACS analysis of the LMX1A-GFP and PITX3-GFP cells from differentiating cultures revealed that $70.2 \%$ and $1.2 \%$ of cells, respectively, expressed GFP (Fig. $1 R, V$ ). Efficiency of the sorting was confirmed by reanalysis of the isolated fractions by flow cytometry, as well as replating of the sorted fractions, and cytochemistry at $6 \mathrm{~h}$ after seeding (Fig. $1 Q, S-U, W-Y$ ). Within the LMX1A-GFP ${ }^{+}$fraction, $>99.2 \%$ of cells expressed GFP, with all GFP cells confirmed to coexpress cardinal vm progenitor markers OTX2 and FOXA2 (data not shown). Within the replated LMX1A-GFP ${ }^{-}$fraction, $10 \%$ of cells were $\mathrm{GFP}^{+}$, a likely reflection of ongoing, asynchronous maturation of the cultures during the period of both sorting and replating (Fig. 1U). Assessment of the sorted PITX3-eGFP cell line confirmed that $>95 \%$ of the replated cells from the positive fraction expressed GFP (Fig. $1 \mathrm{~V}$ ), whereas $\mathrm{GFP}^{+}$cells within the negative fraction were extremely rare (Fig. $1 Y$ ).

\section{Transplanted LMX1a-GFP ${ }^{+}$progenitors, but not PITX3- $\mathrm{GFP}^{+}$DA precursors, restore functional deficits in Parkinsonian rats}

All rats included in the study showed an amphetamine-induced rotational asymmetry $>5$ rotations $/ \mathrm{min}$, before transplantation, that persisted over 24 weeks as a stable motor deficit in ungrafted animals (Fig. 2A,F). As anticipated, and in alignment with hPSCderived vm progenitor grafts by us and others (Kriks et al., 2011; Kirkeby et al., 2012, 2017; Doi et al., 2014; Samata et al., 2016; Niclis et al., 2017a,b), transplants of unsorted cells, from both LMX1a-GFP and PITX3-GFP cell lines, resulted in nearcomplete recovery of rotational asymmetry by 24 weeks. Grafts of LMX1A-GFP ${ }^{+}$progenitors also showed accelerated, complete recovery, evident at 20 weeks. Interestingly, PITX3-GFP ${ }^{-}$, but not PITX3-GFP ${ }^{+}$, cell grafts showed improvements in motor asymmetry at 6 months.

Postmortem analysis showed viable grafts, identified by human PSA-NCAM, in all rats 6 months after implantation (Fig. $2 B-D, G-I)$. Volumetric analysis revealed that LMX1A-GFP ${ }^{+}$ grafts were significantly smaller than both unsorted and LMX1A$\mathrm{GFP}^{-}$cell grafts $\left(3.9 \pm 1.0,8.9 \pm 0.9\right.$, and $10.7 \pm 1.7 \mathrm{~mm}^{3}$, respectively). Grafts of unsorted PITX3-GFP cells were, not surprisingly, comparable with the unsorted LMX1a-GFP cells (9.9 \pm $\left.1.1 \mathrm{~mm}^{3}\right)$, with PITX3-GFP ${ }^{-}$also of similar size $\left(9.3 \pm 1.5 \mathrm{~mm}^{3}\right)$ (Fig. 2E,J). Most evident was the reduced size of the PITX3$\mathrm{GFP}^{+}$cell grafts $\left(0.3 \pm 0.1 \mathrm{~mm}^{3}\right)$. Recognizing that midbrain DA progenitors exit the cell cycle and become postmitotic at a similar time to the onset of PITX3 expression during vm development, combined with knowledge that postmitotic neuroblasts poorly survive transplantation, we speculated that these factors contributed to the small graft size observed. GFP labeling of these grafts confirmed low numbers of PITX3-GFP ${ }^{+}$cells; and while showing a higher density of $\mathrm{GFP}^{+}$cells (data not shown), $\mathrm{TH}^{+} \mathrm{DA}$ neurons were notably sparse ( $591 \pm 84, n=8$ ), highlighting the lack of suitability of this reporter to isolate and enrich DA progenitor/precursors for the purpose of transplantation. 
A

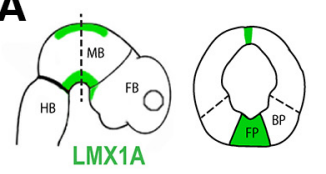

B

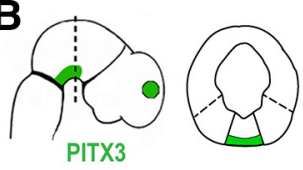

C
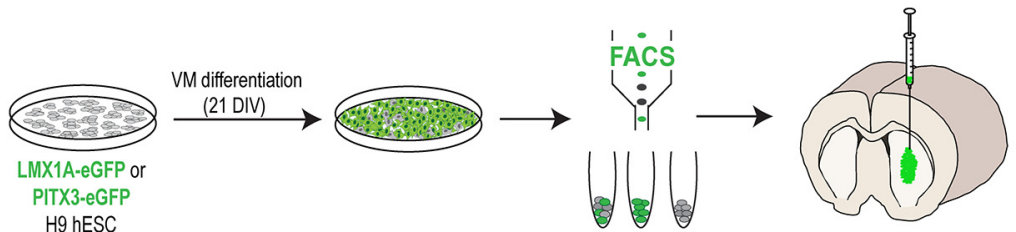

Transplant groups: - Unsorted cells - eGFP+ cell fraction - eGFP- cell fraction
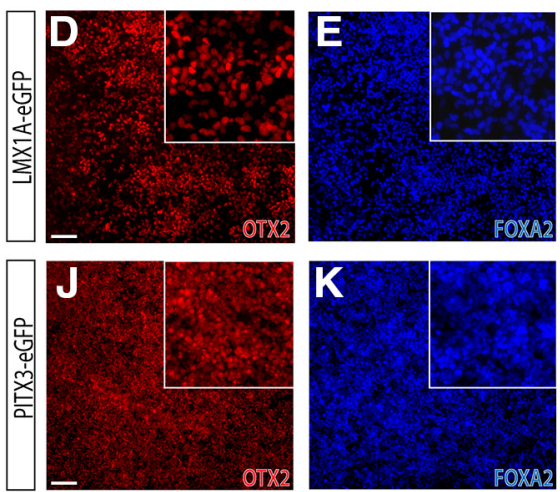

FOXA2
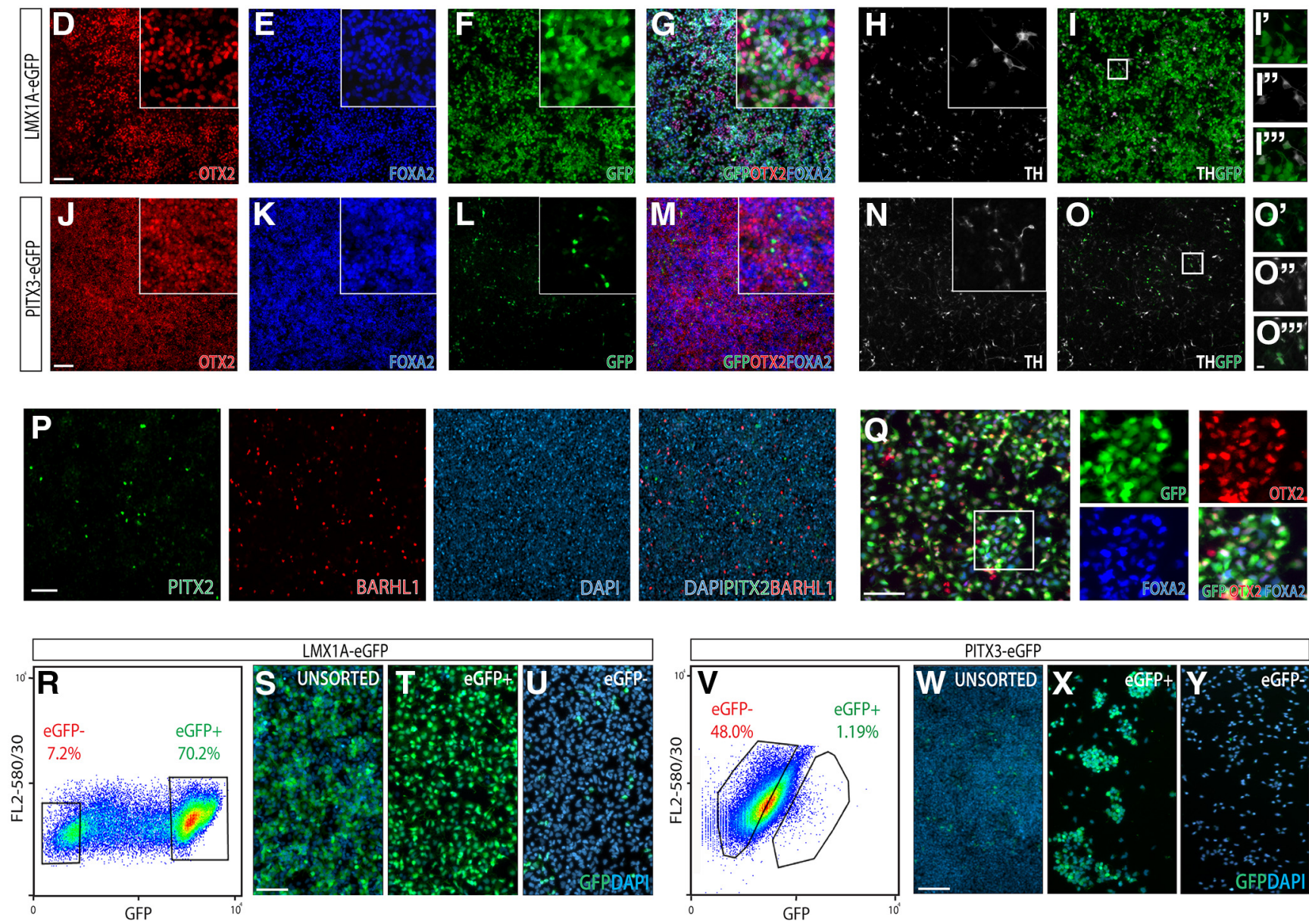

Figure 1. In vitro differentiation and purification of vm progenitors/precursors using LMX1A-eGFP and PITX3-eGFP human ESC reporter lines. $A, B$, Schematic coronal and sagittal views of the developing embryo depicting expression patterns of two key vmDA determinant transcription factors: $(\boldsymbol{A})$ LMX1A and $(\boldsymbol{B})$ PITX3. eGFP was expressed under the promoter for these two transgenes within $\mathrm{H} 9 \mathrm{hESC}$ lines for the purpose of cell sorting. C, Schematic detailing the differentiation, isolation, and transplantation of cells derived from LMX1A-eGFP and PITX3-eGFP hESC reporter lines, and the experimental timeline for in vivo procedures. $\mathbf{D}-\mathbf{G}, \mathbf{J}-\mathbf{M}$, Validation of efficient vm differentiation by coexpression of 0TX2, F0XA2, and eGFP demonstrated robust specification of vm progenitors by 21 DIV.I, $\mathbf{O}$, Maturing dopamine neurons present within the culture at 21 DIV tightly overlapped with eGFP expression. P, Low proportion of PITX2 ${ }^{+}$and BARHL1 ${ }^{+}$cells confirmed that cultures were not of unintended subthalamic identity. $\mathbf{Q}$, Immunohistochemical labeling of LMX1A-GFP ${ }^{+}$progenitors, $6 \mathrm{~h}$ after sorting and replating, showing coexpression of cardinal vmDA progenitor markers OTX2, FOXA2, and LMX1A-GFP. $\boldsymbol{R}, \boldsymbol{V}$, Flow cytometric plots illustrating the gating for enrichment of eGFP ${ }^{+}$and eGFP ${ }^{-}$populations within differentiating cultures at 21 DIV. Successful isolation of specified populations (unsorted, GFP ${ }^{+}$, and GFP ${ }^{-}$) from the $(\boldsymbol{S}-\boldsymbol{U})$ LMX1A-eGFP and $(\boldsymbol{W}-\boldsymbol{Y})$ PITX3-eGFP hPSC line was confirmed by replating of cells and immunohistochemical staining for GFP and DAPI. hESC, Human ESCS. Scale bars: D-0, S-U, W-Y, $200 \mu \mathrm{m} ; \boldsymbol{I}^{\prime}-\boldsymbol{I}^{\prime \prime \prime}, \mathbf{O}^{\prime}-\mathbf{O}^{\prime \prime \prime}, 20 \mu \mathrm{m}$.

Transplantation of LMX1a-GFP ${ }^{+}$vm progenitors enriches for DA neurons that are capable of innervating developmentally appropriate targets in the host brain Reflective of the smaller size of $\mathrm{LMX} \mathrm{A}^{+}$transplants (Fig. 2E), these grafts contained significantly fewer $\left(\mathrm{HNA}^{+}\right)$cells compared with both unsorted or LMX1A-GFP ${ }^{-}$cell grafts (Fig. $3 A$ ). Assessment of GFP staining confirmed the efficiency of the sorting, with $\mathrm{LMX1A}^{-\mathrm{GFP}^{+}}$progenitor grafts showing enrichment of $\mathrm{GFP}^{+}$cells; both total $\mathrm{GFP}^{+}$cells (unsorted:
$162,159 \pm 14,703 ; \mathrm{LMX}^{2} \mathrm{~A}-\mathrm{GFP}^{+}: 225,700 \pm 48,832 ; \mathrm{LMX} 1 \mathrm{~A}-$ $\mathrm{GFP}^{-}: 19,523 \pm 5937$ ) and proportion (Fig. $3 B, E-G$ ). Similar to in vitro observations, transplants of LMX1A-GFP ${ }^{-}$progenitors resulted in grafts containing pockets of GFP ${ }^{+}$cells (Fig. 3G), likely reflective of the asynchronous maturation of younger progenitors adopting an $\mathrm{LMXla}^{+}$fate after the time of sorting and implantation.

Unsurprisingly, the total number of $\mathrm{TH}^{+}$DA neurons quantified within $\mathrm{LMX}^{\mathrm{A}} \mathrm{A}-\mathrm{GFP}^{+}$and unsorted grafts was not signifi- 

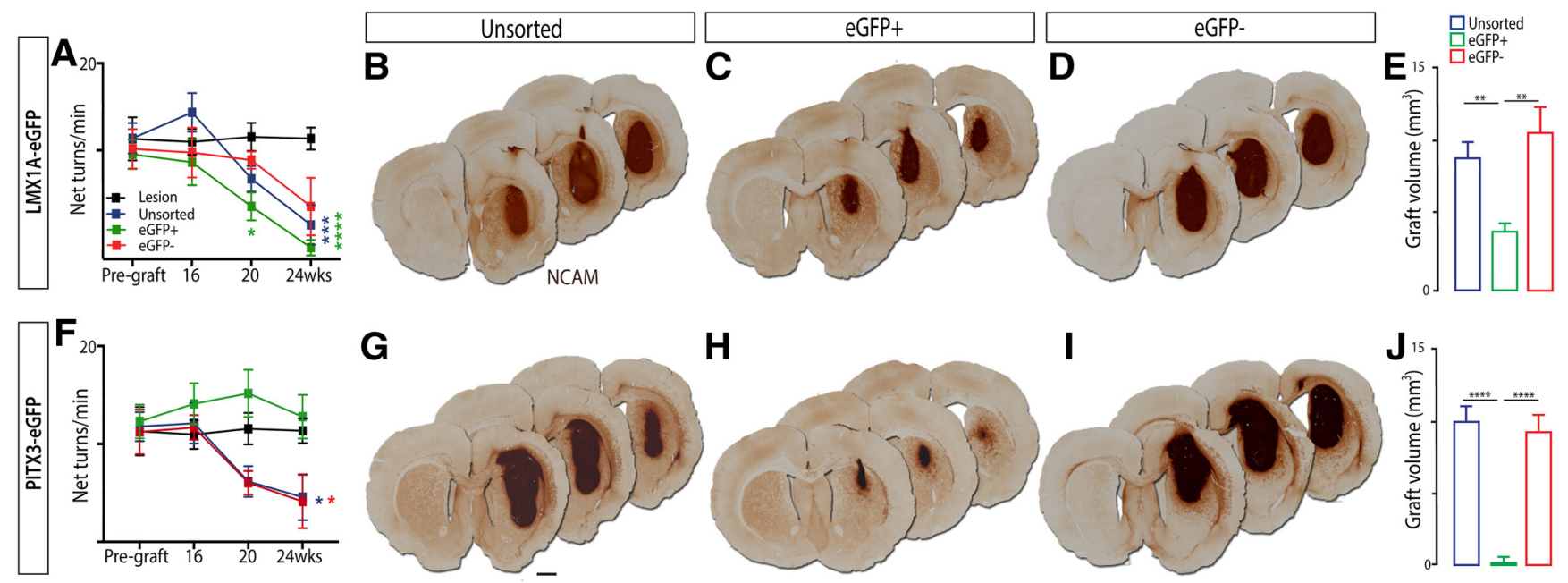

Figure 2. LMX1a-eGFP ${ }^{+}$progenitors, but not PITX3-eGFP ${ }^{+}$precursors, show engraftment and amelioration of behavioral deficits in 6-0HDA-lesioned rats. $A$, Transplantation of LMX1A-eGFP ${ }^{+}$ progenitors (green) resulted in an accelerated correction of rotational asymmetry in 6-OHDA athymic rats, compared with grafts of unsorted (blue) and eGFP ${ }^{-}$vm progenitors (red) (two-way ANOVA with Dunnett's correction for multiple comparisons relative to 6-0HDA group, $F_{(3,88)}=9.131, p<0.0001, n=6-8 /$ group). $\boldsymbol{B}-\boldsymbol{D}$, Human-specific NCAM enables visualization of the LMX1A progenitor grafts within the host striatum. $E$, Quantitative assessment of LMX1A-eGFP progenitor grafts highlighting the smaller size of grafts derived from eGFP ${ }^{+}$progenitors (one-way ANOVA with Tukey's posthoc test, $F_{(2,14)}=9.8, p=0.0022, n=6-8 /$ group). $F$, Grafting of PITX3-eGFP ${ }^{-}$and unsorted, but not PITX3-eGFP ${ }^{+}$cells, corrected gross motor deficits in 6-0HDA rats (two-way ANOVA, $F_{(3,120)}=3.188, p=0.0263, n=6-8 /$ group). $G-I$, Coronal sections of the rat brain showing large viable grafts 6 months after transplantation of unsorted and PITX-eGFP ${ }^{-}$cells. PITX3-eGFP ${ }^{+}$DA precursors showed poor survival and engraftment following transplantation, $(J)$ confirmed by significantly reduced graft volume (one-way ANOVA, $F_{(2,16)}=30.61, p=<0.0001$, $n=6-8$ /group). Data are mean \pm SEM. Scale bars: $\boldsymbol{B}-\boldsymbol{D}, \mathbf{G}-\mathbf{I}, 1 \mathrm{~mm} .{ }^{*} p<0.05,{ }^{* *} p<0.01,{ }^{* *} p<0.001,{ }^{* * *} p<0.0001$.

cantly different $(19,310 \pm 2828$ and $23,318 \pm 3697)$ (Fig. $3 C, H, I)$. Most striking, however, was the density and distribution of these neurons (Fig. 3D, $H-J$ ). Compared with unsorted grafts, which contained a heterogeneous distribution of TH neuron, including areas of high $\mathrm{TH}^{+}$cell numbers $\left(\right.$Fig. $\left.3 \mathrm{H}^{\prime}\right)$ and areas of low $\mathrm{TH}^{+}$(Fig. $3 \mathrm{H}^{\prime \prime}$ ), transplants of LMX1A-GFP ${ }^{+}$progenitors resulted in a dense, homogeneous network of $\mathrm{TH}^{+}$cells (with $>2$-fold increase in density) that minimally displaced host striatal tissue (Fig. 3I, $I^{\prime}, I^{\prime \prime}$ ). Reflective of the selection for non-VM progenitors, LMX1A-GFP ${ }^{-}$grafts showed sparse $\mathrm{TH}^{+}$DA neurons (Fig. 3J). Interestingly, upon analysis of the replated LMXla-GFP ${ }^{-}$fraction in vitro, a number of $\mathrm{GFP}^{-} / \mathrm{TH}^{+}$cells were observed, indicative of a non-vmDA cell population (Fig. $3 M$, arrowhead) that was not seen in the replated LMX1A-GFP ${ }^{+}$ cell fraction (Fig. $3 L$ ). Within LMX1A ${ }^{+}$grafts, $79.2 \pm 8.2 \%$ of

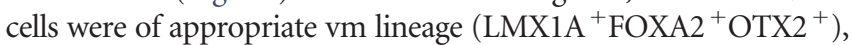
of which $\sim 7 \%$ became mature $\left(\mathrm{TH}^{+}\right)$DA neurons, 2 -fold higher than unsorted grafts.

To confirm that selection of LMX1A-GFP ${ }^{+}$progenitors did not affect the propensity (or proportion) of DA neurons to adopt an A9 substantia nigra pars compacta-like or A10 ventral tegmental area-like fate, we assessed the phenotype of $\mathrm{TH}^{+}$cells within the grafts. As previously reported (Grealish et al., 2014; Niclis et al., 2017b), $>85 \% \mathrm{TH}^{+}$cells in all grafts showed expression of the A9 and/or A10 proteins, GIRK2 and calbindin, respectively, with no significant difference between any of the transplant groups (Fig. $3 K, L$ ). In alignment with postmortem assessment of the human midbrain (Reyes et al., 2012), the majority of $\mathrm{TH}^{+}$ cells coexpressed GIRK2, alone or together with calbindin, while few $\mathrm{TH}^{+}$neurons expressed only calbindin ${ }^{+}$.

Necessary for the functional efficiency of vmDA grafts is their capacity to send afferent projections to the host striatum. In accordance with the similar numbers of TH neurons, LMX1A ${ }^{+}$ grafts showed comparable density of innervation of the dorsal striatum and cortex to grafts of unsorted progenitors (Fig. 4A$D)$. In contrast, $\mathrm{LMX1A-GFP}^{-}$progenitor grafts poorly inner- vated these motor function-associated nuclei (Fig. 4A,B,E), rather showing increased connectivity with nondopaminergic target nuclei, inclusive of the periventricular thalamic nucleus and hippocampus (Fig. $4 F-L$ ).

While we confirmed a low proportion of STN progenitors within our cultures before transplantation (a rostral vm population known to express LMX1A) (Kee et al., 2017), residing within the appropriately specified, more caudal, domain of the developing vm are other nondopaminergic LMX1A-expressing progenitors. With $<9 \%$ of the $\mathrm{GFP}^{+}$cells expressing $\mathrm{TH}$ within $\mathrm{GFP}^{+}$progenitor grafts (or 19\%, as a proportion of total implanted cells), we sort to perform a more detailed assessment of the composition of these grafts, focusing on neural specification. All neural lineages were present within the transplants with $>55 \%$ of $\mathrm{HNA}^{+}$cells coexpressing the postmitotic neuronal marker NeuN within the GFP ${ }^{+}$progenitor grafts, significantly greater than unsorted cell grafts (Fig. $3 P-R$ ). In contrast, sorting reduced the contribution of $\mathrm{APC}^{+}$mature oligodendrocytes (Fig. 3S), and no change was observed in the density of GFAP ${ }^{+}$ astrocytes (Fig. 3T). More detailed assessment of neuronal subpopulations revealed that $\mathrm{DBH}^{+}, \mathrm{ChAT}^{+}$, and $\mathrm{GAD}^{+} 7^{+}$cells were noticeably rare within the $\mathrm{GFP}^{+}$grafts (data not shown). Staining against vascular proteins targeted to the host (RECA1) versus broader/non-species-specific (Claudin V), revealed that the sparse density of vessels within the grafts was of host, and not graft, origin (data not shown). While quantitative assessment of $\mathrm{GFAP}^{+}$astrocytes was not feasible (due to the dense network of staining and inability to resolve graft from host cells with certainty), there was at least some evidence of $\mathrm{HNA}^{+}$cells failing to adopt any of the three neural lineages. Such observations reflect our incomplete knowledge of the lineage trajectories of the $\mathrm{LMX} 1 \mathrm{~A}^{+} / \mathrm{FOXA}_{2}{ }^{+} / \mathrm{OTX} 2^{+}$progenitor; and while recently recognized as having the capacity to give rise to mature astrocytes in vitro (Holmqvist et al., 2015), other populations may remain to be identified. 

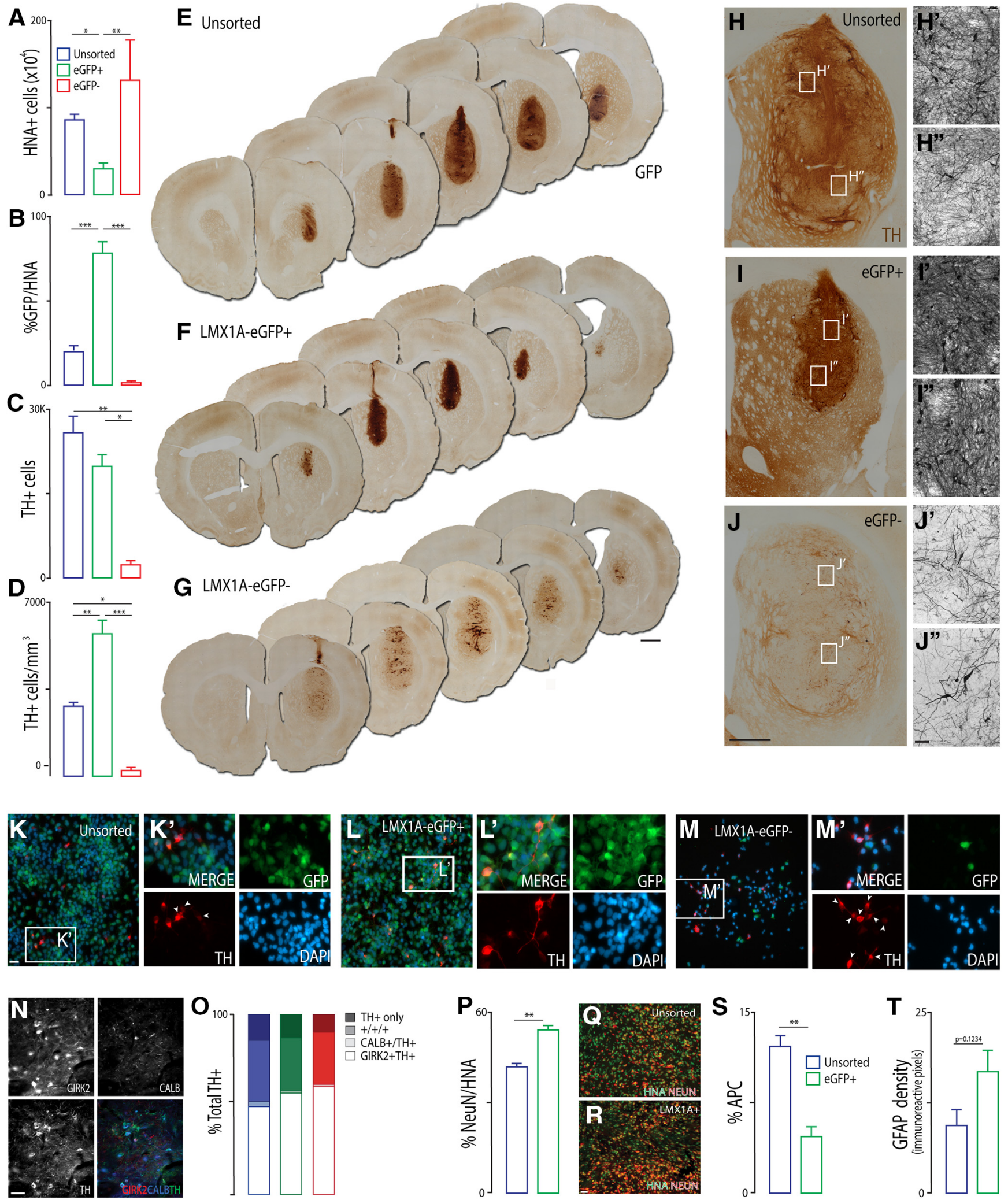

Figure 3. Transplants of LMX1A-eGFP ${ }^{+}$progenitors result in discrete, homogeneous grafts with enriched density of dopaminergic neurons of maintained A9/A10 subtype specification. $A$, Quantitative assessments of transplants revealed significantly fewer HNA ${ }^{+}$cells (one-way ANOVA, $F_{(2,14)}=9.445, p=0.0025 ; n=6-8 /$ group), and (B) enrichment of GFP-expressing cells within grafts of LMX1A-GFP ${ }^{+}$progenitors, compared with both unsorted and LMX1A-GFP- (one-way ANOVA, $F_{(2,13)}=42.15, p<0.0001 ; n=6-8 /$ group$) . ~^{-}$, TH ${ }^{+}$cell number did not differ from unsorted progenitor grafts (one-way ANOVA, $F_{(2,12)}=8.445, p=0.0051 ; n=6-8 /$ group). $\boldsymbol{D}$, TH density was significantly elevated within LMX1A-GFP ${ }^{+}$progenitor grafts, compared with transplants of unsorted and LMX1A-GFP ${ }^{-}$cells (one-way ANOVA, $F_{(2,12)}=19.68, p=0.0002 ; n=6-8 /$ group). Representative overview of $(\boldsymbol{E})$ unsorted, $(\boldsymbol{F})$ LMX1A-GFP $^{+}$, and $(\boldsymbol{G})$ LMX1A-GFP $^{-}$ grafts showing GFP expression. $\boldsymbol{H}-\boldsymbol{J}$, Photomicrograph showing the distribution and density of $\mathrm{TH}^{+}{ }^{+}$cells within grafts derived from unsorted, LMX1A-GFP ${ }^{+}$, and LMX1A-GFP ${ }^{-}$progenitors. $\boldsymbol{H}^{\prime}, \boldsymbol{H}^{\prime \prime}$, The heterogeneity in TH cell density seen in unsorted grafts, by comparison with $\left(I^{\prime}, I^{\prime \prime}\right)$ the dense homogeneous distribution observed in LMX1A-GFP ${ }^{+}$grafts. $J^{\prime}$, Transplants of LMX1A-GFP ${ }^{-}$ progenitors showed few $\mathrm{TH}^{+}$cells scattered throughout the transplant. $\boldsymbol{K}-\boldsymbol{M}$, Representative images of unsorted $(\boldsymbol{K}), \mathrm{LMX}^{\mathrm{A}} \mathrm{A}-\mathrm{GFP}^{+}(\boldsymbol{L})$, and LMX1A-GFP- ${ }^{-}$(Figure legend continues.) 
A

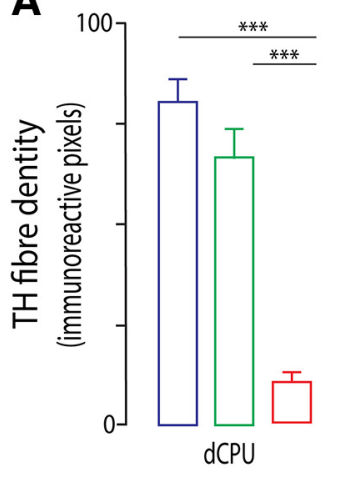

B

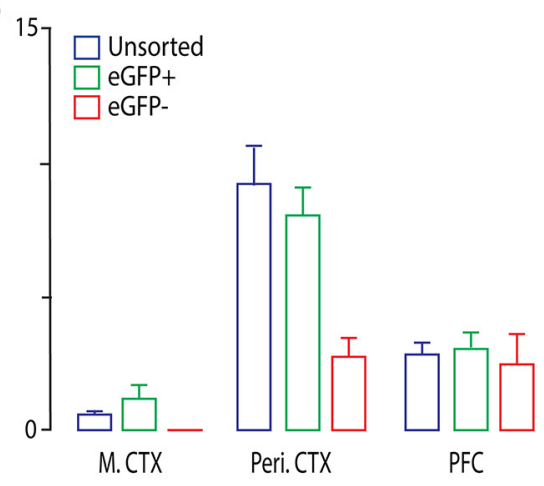

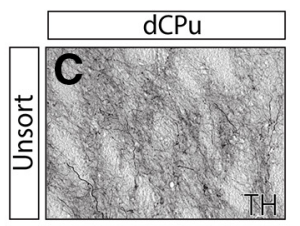
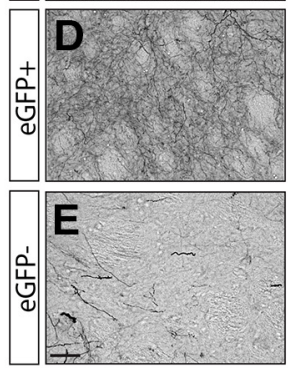

$\mathbf{F}$
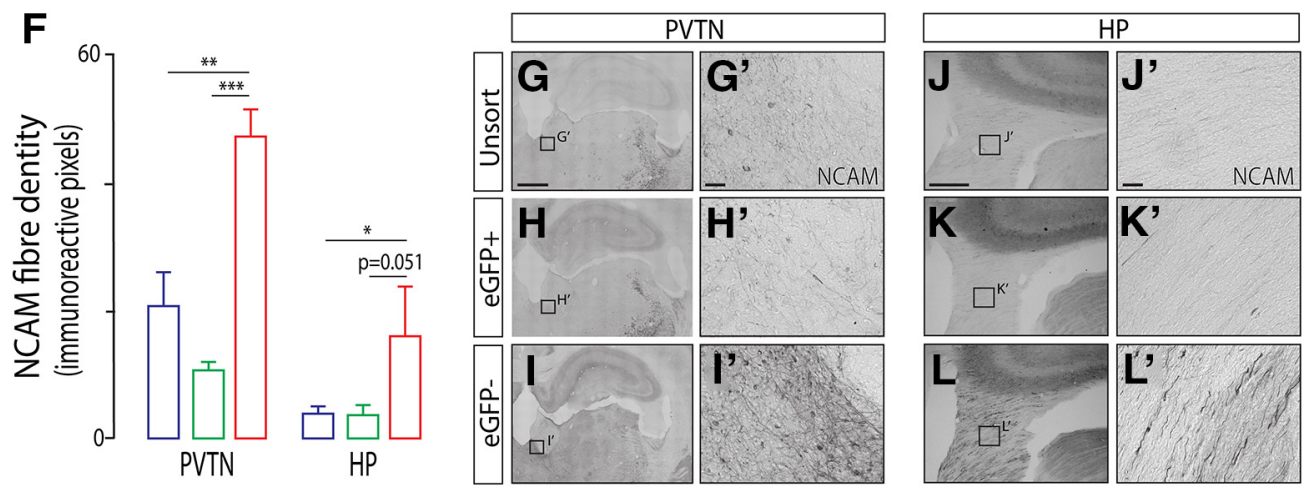

Figure 4. Grafts containing $\mathrm{LMX1A}^{-}$cells preferentially innervate nondopaminergic targets. $A$, Quantitative assessment of $\mathrm{TH}^{+}$fiber density in the dorsal caudate-putamen (dCPU, one-way ANOVA, $F_{(2,10)}=32.77, p<0.0001 ; n=4-6 /$ group) and $(\boldsymbol{B})$ cortex, inclusive of the motor cortex (M.CTX), perirhinal cortex (Peri.CTX), and PFC. C $\boldsymbol{E}$, High-power images illustrating the increased density of $\mathrm{TH}^{+}$fibers in the striatum of unsorted and $\mathrm{LMX1A-GFP}{ }^{+}$cell grafts compared with grafts derived from LMX1A-GFP ${ }^{-}$progenitors. $F$, Quantification of human NCAM ${ }^{+}$fiber density in the periventricular thalamic nucleus (PVTN, one-way ANOVA, $F_{(2,13)}=16.75, p=0.0003 ; n=4-6 /$ group) and fimbria of the contralateral hippocampus (HP, one-way ANOVA, $F_{(2,13)}=4.308, p=$ $0.0367 ; n=4-6 /$ group) from grafted animals. $\mathbf{G}-\mathbf{I}$, Representative photomicrographs showing increased density of NCAM ${ }^{+}$fibers within the PVTN and $(J-L) H P$ in animals transplanted with LMX1A-GFP $^{-}$progenitors, compared with grafts of unsorted or LMX1A-GFP ${ }^{+}$cells, demonstrative of off-target innervation. Data are mean \pm SEM. Scale bars: $C-E, 50 \mu \mathrm{m} ; \mathbf{G}-I, J-L, 200 \mu \mathrm{m}$; $G^{\prime}-I^{\prime}, J^{\prime}-L^{\prime}, 20 \mu \mathrm{m} .{ }^{*} p<0.05,{ }^{* *} p<0.01,{ }^{* * *} p<0.001$.

\section{Isolation of LMX1A-GFP ${ }^{+}$progenitors reduces proliferating cell populations and eliminates serotonergic neurons from hPSC-derived vm grafts}

Reflective of the general efficiency of the in vitro differentiation of the hPSC LMX1A-GFP cell line, and the in vivo maturation of the implanted progenitors, no gross neural overgrowths or tumors were observed in any grafted animals after 26 weeks. The increase

\section{$\leftarrow$}

(Figure legend continued.) (M) progenitors $6 \mathrm{~h}$ after sorting and replating showing the coexpression of GFP with TH. While $\mathrm{TH}^{+} \mathrm{GFP}{ }^{+}$cells numbers were low in all replated cultures, reflective of the stage of differentiation (21 DIV), the LMX1A-GFP ${ }^{-}$cultures showed a high proportion of $\mathrm{TH}^{+} \mathrm{GFP}^{-}$cells (white arrowheads), indicative of an earlier-born, non-vm, dopaminergic or adrenergic/noradrenergic cell population. $\boldsymbol{N}$, Representative images showing the coexpression of TH together with GIRK2 and/or calbindin, indicative of A9- and A10-like specification. $\mathbf{0}$, Quantification of $\mathrm{TH}^{+}$neurons that showed coexpression of GIRK2 and/or calbindin confirmed that transplanted $\mathrm{LMX} \mathrm{A}-\mathrm{GFP}^{+}$progenitors retained their propensity to form mature vmDA neurons of both A9 and A10 phenotype. $\boldsymbol{P}$, Isolation of LMX1A-GFP ${ }^{+}$cells resulted in grafts significantly enriched with $\mathrm{NeuN}^{+}$neurons, compared with unsorted vm progenitor grafts (unpaired $t$ test, $t=7.76, \mathrm{df}=4, p=0.0015 ; n=3$ or 4/group). $\mathbf{Q}, \boldsymbol{R}$, Representative photomicrographs of NeuN-immunolabeled neurons within an unsorted and GFP ${ }^{+}$graft. $S$, The proportion of $\mathrm{APC}^{+}$mature oligodendrocytes was significantly reduced in LMX1A-GFP ${ }^{+}$ grafts (unpaired $t$ test, $t=5.32, \mathrm{df}=4, p=0.006 ; n=3$ or 4/group), whereas the relative contribution of $(\boldsymbol{T}) \mathrm{GFAP}^{+}$astrocytes remained unchanged (unpaired $t$ test; $n=3$ or $4 /$ group). Data are mean \pm SEM. Scale bars: $\boldsymbol{E}-\boldsymbol{J}, 1 \mathrm{~mm} ; \boldsymbol{H}^{\prime}-\boldsymbol{J}^{\prime \prime}, \boldsymbol{N}, 50 \mu \mathrm{m} .{ }^{*} p<0.05,{ }^{* *} p<0.01$, ${ }^{* * *} p<0.001$ in total cells, from 100,000 implanted cells to a total of 836,883 \pm 54,083 cells within the unsorted grafts at 6 months, reflects the proliferative capacity of the progenitors at the time of implantation. While the majority of cells adopted a postmitotic fate, a small dividing population remained present at 26 weeks, identified by doublecortin and KI67 staining within unsorted and LMX1A-GFP $^{-}$progenitor grafts. Supportive of the smaller graft volumes observed, transplants of LMX1A-GFP ${ }^{+}$progenitors contained significantly fewer dividing cells $(0.18 \pm 0.01 \%$ $\mathrm{KI} 67^{+} / \mathrm{HNA}^{+}$; Fig. $5 A-D$ ).

Finally, we assessed grafts for the presence of $5-\mathrm{HT}^{+}$serotonergic neurons, a population known to contribute to the development of graft-induced dyskinesias in preclinical and clinical studies (Carlsson et al., 2007; Politis et al., 2011). Transplants of LMX1A-GFP $^{+}$progenitors eliminated serotonergic neurons, with just 2 grafts containing a single 5-HT-immunoreactive neuron, compared with the significantly higher numbers observed in both unsorted $(7346 \pm 1382)$ and LMX1A-GFP ${ }^{-}(2168 \pm 359)$ grafts, and consequently also the proportion of serotonergic to dopaminergic neurons (DA:5-HT, Fig. 5E-I), a recognized contributing factor in the incidence of induced dyskinesias (García et al., 2011; Politis et al., 2011).

Demonstrating the utility and reproducibility of sorting for LMX1A-expressing progenitors, we repeated FACS isolation and transplantation into 6-OHDA-lesioned athymic mice. Again, we 
showed smaller grafts with enrichment for both GFP and TH neurons (Fig. 6A-I). $\mathrm{TH}^{+}$DA neuronal density within the grafts was surprisingly similar to the rats, with significant increases in density (and homogeneity) observed in the LMX1A$\mathrm{GFP}^{+}$, compared with unsorted and LMX1A-GFP $^{-}$grafts (Fig. 6F,I). No 5-HT neurons were observed in grafts of ${\mathrm{LMX} 1 \mathrm{~A}-\mathrm{GFP}^{+}}^{+}$progenitors (Fig. $6 J-L$ ).

\section{Discussion}

Heterogeneity in graft composition following the implantation of hPSC-derived vm progenitors presents ongoing concerns for the safety and functional predictability of these cells for future clinical application in Parkinson's disease. For these reasons, efforts to improve the homogeneity of the donor preparation, and thereby graft compositions, are of significant importance. In this study, we developed two GFP-expressing reporter lines, LMX1A-GFP and PITX3-GFP, to enable the selective isolation of correctly specified vm progenitors/precursors from differentiating hPSC cultures, at differing developmental stages. We demonstrate that transplants of LMX1A-GFP-expressing progenitors result in discrete grafts with increased density of DA neurons and greater, target-appropriate innervation, resulting in accelerated functional recovery of motor symptoms, compared with unsorted progenitor grafts. In contrast, transplants of PITX3-GFP ${ }^{+}$cells resulted in notably small grafts with low $\mathrm{TH}^{+}$DA neuron yields. While PITX3-GFP populations have been successfully isolated from mouse ESC cultures (Hedlund et al., 2008; Ganat et al., 2012), the present findings are in accordance with fetal tissue grafting studies using PITX3-GFP embryos; recognizing the vulnerability of this postmitotic population to shear trauma during periods of culture detachment/dissociation, FACS, and implantation (Jönsson et al., 2009). Such findings highlight the need to identify a developmentally relevant differentiation stage of hPSC-derived vm progenitors that are sufficiently specified and also amenable for grafting, as has been achieved for rodent and human fetal tissue (Freeman et al., 1995; Torres et al., 2007; Bye et al., 2012) as well as mouse PSCs (Ganat et al., 2012).

In addition to the significance of enriching for DA neurons within grafts is the concomitant capacity to eliminate poorly specified and high proliferative populations that may lead to overgrowth or graft-induced dyskinesias. While incorrectly specified cells are in the minority in vitro, due to advances in hPSC neuronal differentiation protocols (Kriks et al., 2011; Niclis et al.,
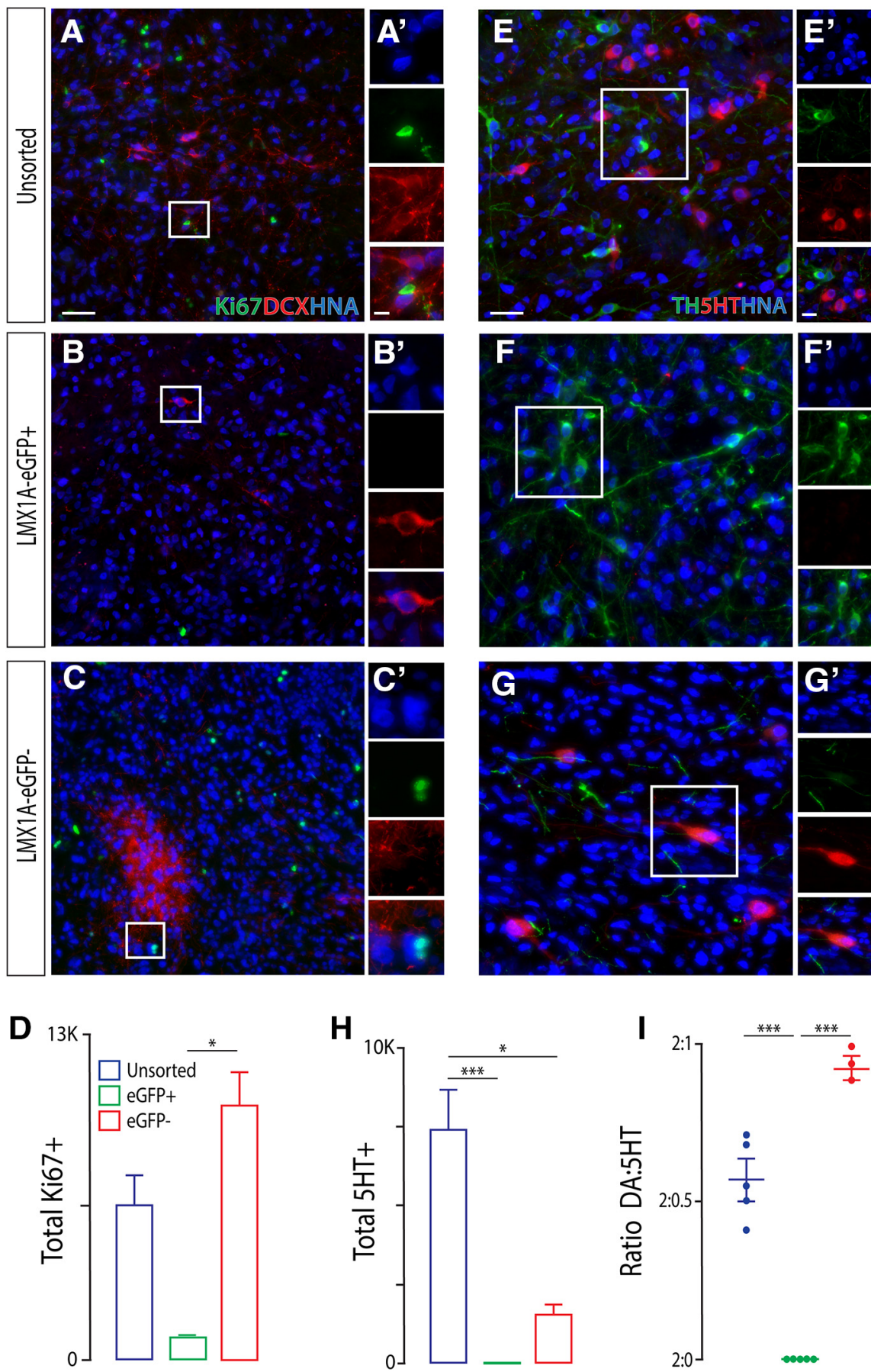

Figure 5. Transplantation of LMX1A-GFP ${ }^{+}$progenitors reduces the presence of proliferating cells and eliminates incorrectly specified serotonergic neurons within grafts. $A-C$, Immunohistochemical labeling of transplants with HNA (blue), DCX (red), and $\mathrm{KI} 67$ (green), illustrating the reduction in proliferative progenitors at 6 months within transplants derived from LMX1A-GFP ${ }^{+}$. $D$, Quantification of Ki67 ${ }^{+}$cells within the LMX1A-eGFP cell grafts (one-way ANOVA, $F_{(2,6)}=8.248, p=0.019 ; n=4-6 /$ group). $\boldsymbol{E}-\boldsymbol{G}$, Representative photomicrographs showing dopaminergic $\left(\mathrm{TH}^{+}\right.$, green), serotonergic $\left(5-\mathrm{HT}^{+}{ }^{+}\right.$, red), and HNA immunolabeling within transplants of unsorted, GFP ${ }^{+}$and GFP ${ }^{-}$progenitor grafts. $\boldsymbol{H}$, Quantification of 5 - $\mathrm{HT}^{+}$cells (one-way ANOVA, $F_{(2,13)}=17.26, p=0.0003, n=4-6 /$ group), and (I) ratio of dopaminergic to serotonergic (DA:5-HT) neurons within the transplants (one-way ANOVA, $F_{(2,11)}=57.19, p=<0.0001 ; n=4-6 /$ group), confirmed the near-complete elimination of the dyskinetic-contributing cell population from grafts derived from LMX1A-GFP ${ }^{+}$progenitors. Data are mean \pm SEM. Scale bars: $A-C, E-G, 50 \mu \mathrm{m} ; A^{\prime}-C^{\prime}, E^{\prime}-G^{\prime}, 20 \mu \mathrm{m} .{ }^{*} p<0.05,{ }^{* *} p<0.001$.

2017a), these populations have a propensity for expansion following implantation and/or can impart negative effects on transplant function. On average, studies have reported a yield of $5 \%-6 \% \mathrm{TH}^{+}$DA cells in the grafts per 100,000 cells implanted (Kriks et al., 2011; Kirkeby et al., 2012, 2017; Doi et al., 2014; 

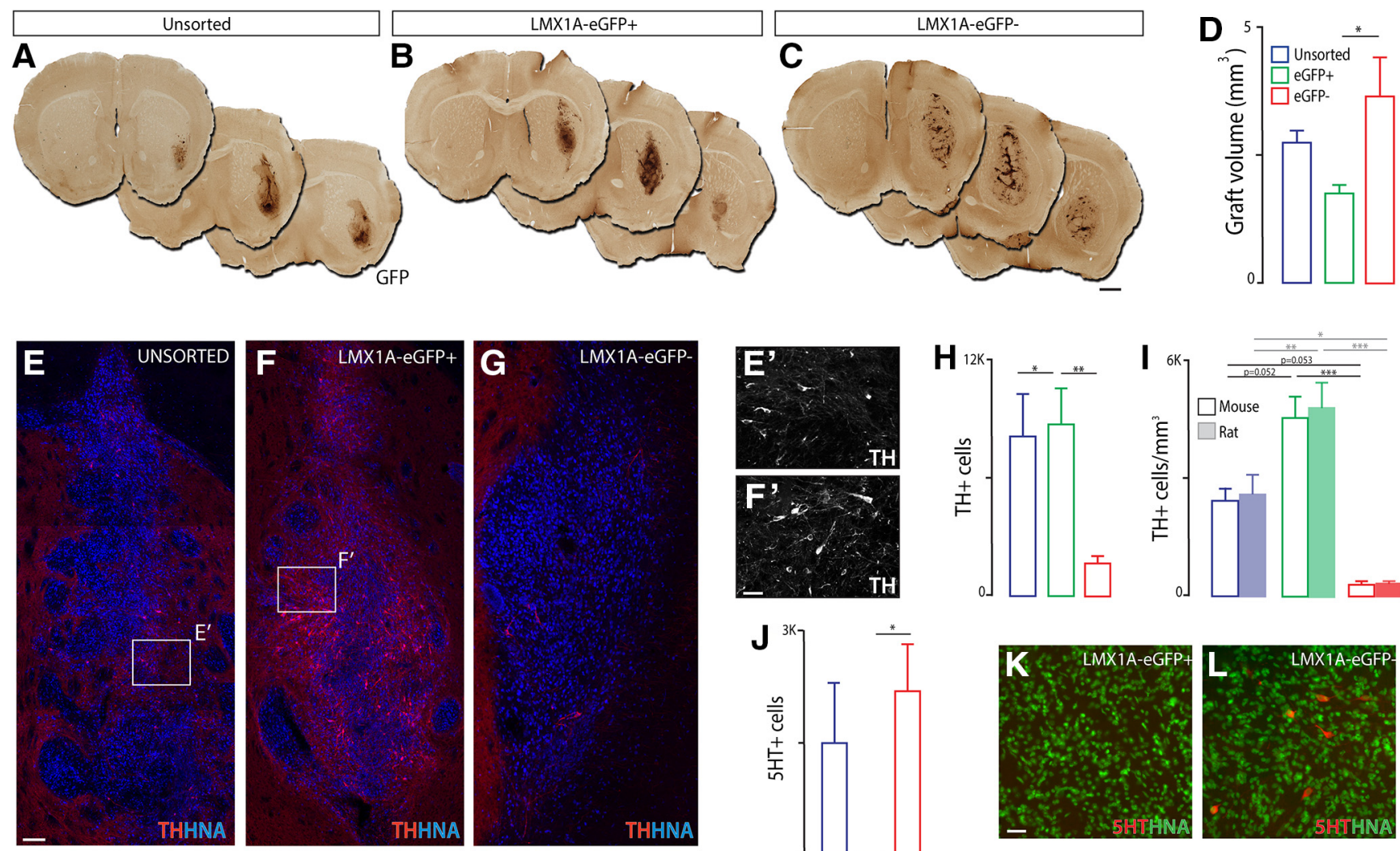
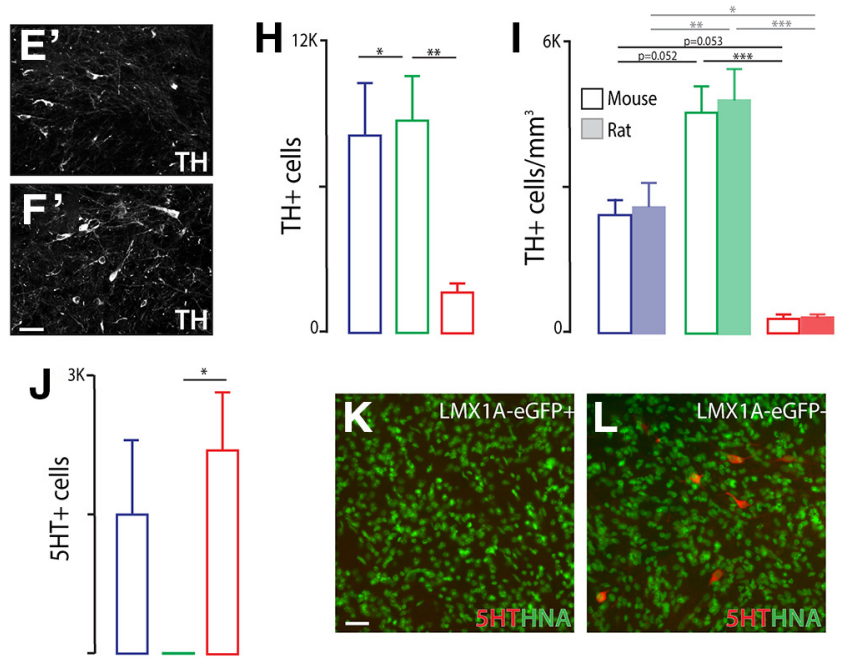

Figure 6. Transplantation of LMX1A-GFP ${ }^{+}$progenitors shows high reproducibility across repeated rodent transplantation studies. Photomicrographs showing GFP immunohistochemical

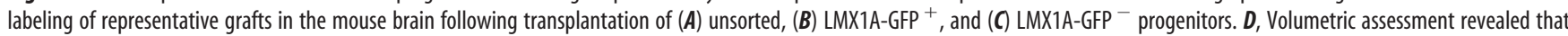

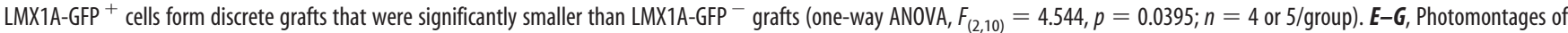
representative grafts in mice illustrating $\mathrm{TH}^{+}$(red) and $\mathrm{HNA}^{+}$(blue) labeling. $\boldsymbol{F}^{\prime}$, Enrichment of dopaminergic neurons within $\mathrm{LMX1A-GFP}{ }^{+}$transplants. $\boldsymbol{H}_{\text {, Quantification of total TH }}^{+}$cells (one-way ANOVA, $F_{(2,15)}=7.566, p=0.0053, n=4$ or 5/group), and ( $\left.I\right)$ TH cell density (rats: one-way ANOVA, $F_{(2,12)}=19.68, p=0.0002, n=4-6 /$ group; mice: one-way ANOVA, $F_{(2,9)}=18.91$, $p=0.0006, n=4$ or 5/group) within grafts derived from unsorted, LMX1A-GFP ${ }^{+}$, and LMX1A-GFP ${ }^{-}$progenitors and transplanted into mice (open bars) and rats (closed bars). J, Quantification of $5-\mathrm{HT}^{+}$cells within LMX1A progenitor grafts in mice (one-way ANOVA, $F_{(2,9)}=9.816, p=0.0055, n=4$ or $5 /$ group), showing reproducible elimination of serotonergic neurons (as seen also in transplants in rats). $\boldsymbol{K}, \boldsymbol{L}$, High-power images highlighting the elimination of 5-HT neurons in LMX1A-GFP ${ }^{+}$grafts. Data are mean \pm SEM. Scale bars: $\boldsymbol{A}-\boldsymbol{C}, 1 \mathrm{~mm} ; \boldsymbol{E}-\boldsymbol{G}, 500 \mu \mathrm{m} ; \boldsymbol{E}^{\prime}-\boldsymbol{F}^{\prime}, 20 \boldsymbol{\mu m} ; \boldsymbol{K}$, $L, 50 \mu \mathrm{m} .{ }^{*} p<0.05,{ }^{* *} p<0.01,{ }^{* * *} p<0.001$.

Samata et al., 2016; Niclis et al., 2017b). This yield, however, is notably lower when expressed as a proportion of the total number of cells within the graft, given the proliferation of the vm progenitors following implantation (Samata et al., 2016; Niclis et al., 2017b). The impact of the incorrectly specified cells has been most evidently demonstrated by Niclis et al. (2017b), where, despite $>85 \%$ correctly specified vmDA progenitors present in culture (confirmed by FOXA2/OTX2/LMX1A coexpression) and maintained expression in the majority of cells observed 4 weeks after implantation, by 28 weeks graft volume increased 20 -fold with no increase in $\mathrm{TH}^{+}$cell numbers (Niclis et al., 2017a,b). Also recognizing this challenge, Kirkeby et al. (2017) describe the lack of predictability of grafting outcomes across many $(>30)$ hPSC vm differentiations, despite consistent ( $>80 \%$ FOXA2/ $\mathrm{OTX}^{+}$) in vitro specification of the progenitors before transplantation. In the present study, the discrete size of LMX1A$\mathrm{GFP}^{+}$grafts, lack of proliferating cells, and increased proportion of $\mathrm{TH}^{+}$DA neurons observed at 6 months are a significant improvement and a stark contrast to grafts of the LMX1A-GFP ${ }^{-}$ populations. Together, these results highlight the dramatic impact of eliminating poorly specified and/or proliferating cells and underline the necessity for vm progenitor enrichment before grafting.

The benefit of eliminating poorly specified neural progenitors can be further appreciated in the context of recent work high- lighting the level of off-target innervation made by hPSC-derived vm progenitor grafts (Niclis et al., 2017b). While earlier studies were unable to specifically track the relative contribution of dopaminergic and nondopaminergic innervation from the graft into the host brain, Niclis et al. (2017b) made use of the PITX3eGFP reporter line to demonstrate that the dopaminergic neurons within the grafts innervated developmentally appropriate host targets, inclusive of the striatum and cortex. However, the vast majority of graft-derived fiber outgrowth was nondopaminergic, resulting in extensive axonal growth through permissive white matter tracts and significant innervation of off-target nuclei (Niclis et al., 2017b). Supporting these findings, here we demonstrate that the dopamine-enriched LMX1A-GFP ${ }^{+} \mathrm{vm}$ progenitor grafts maintain preferential innervation of appropriate dopaminergic target nuclei with low-level innervation of other nuclei. In contrast, LMX1A-GFP ${ }^{-}$grafts show enhanced innervation of target nuclei that typically have low midbrain dopaminergic innervation, such as the thalamus and hippocampus, which carry unknown consequences.

Elimination of serotonergic neurons, a population of cells that have been shown to contribute to graft-induced dyskinesias in both preclinical and clinical studies, is an additional requisite in the refinement of hPSC grafting targeted for Parkinson's disease (Carlsson et al., 2007; Politis et al., 2011). These undesirable neu- 
rons, generated in the ventral basal plate of the rostral hindbrain, reside outside the region of expression of LMX1A (Fig. 1B) (Mishima et al., 2009; Kirkeby et al., 2012), in contrast to other target genes, such as FolR1 and Corin, which have been used to enrich for DA progenitors (Doi et al., 2014; Gennet et al., 2016). Here we demonstrate the capacity to eliminate $5 \mathrm{HT}^{+}$serotonin cells from within human LMX1A-GFP ${ }^{+}$progenitor grafts, in both rat and mouse grafting studies. Of note, the significant reduction in $5-\mathrm{HT}^{+}$neurons observed in grafts of LMX1A-GFP ${ }^{-}$ progenitors (Fig. $5 H$ ), compared with the unsorted cell grafts, reflects the stringency of the FACS gating used within the study (Fig. 1R), with an estimated $23 \%$ of cells excluded from both the $\mathrm{GFP}^{+}$or $\mathrm{GFP}^{-}$pool.

The importance of cell-sorting approaches for clinical applications is by no means a new concept. Most evidently demonstrated, and clinically translated, has been the purification of hematopoietic stem cells from blood for the treatment of, for example, some blood-related cancers and autoimmune diseases. Preclinical studies focused on the isolation of vm progenitors from differentiating hPSC cultures is also not novel in the context of improving the safety and predictability of transplants for Parkinson's disease. While a number of rodent studies successfully isolated vm progenitors, using FACS or magnetic beads, targeting intracellular and extracellular proteins, respectively (Fukuda et al., 2006; Thompson et al., 2006; Hedlund et al., 2008; Jönsson et al., 2009; Ganat et al., 2012; Nefzger et al., 2012; Bye et al., 2015), isolation from hPSC cultures has been met with variable success.

In a number of studies, candidate markers used for selection purposes have been recognized as too broad in their expression to selectively isolate vm progenitors, such as the pan-neuronal marker CD56/NCAM (Pruszak et al., 2007), ventral floorplate marker FOXA2 (Aguila et al., 2014), vm floor and basal plate markers integrin associated protein and LMRT1 (Aguila et al., 2014; Samata et al., 2016; Lehnen et al., 2017), as well as vm/hindbrain marker CORIN (Doi et al., 2014). Studies also conducted sorting early in the differentiation (due to timing of expression of the selectable protein) and required extended periods of replating before transplantation (Doi et al., 2014; Samata et al., 2016; Lehnen et al., 2017). Such replating steps undermine initial purification and may introduce downstream variability in cell composition at the time of grafting, and have been shown to result in poor $\mathrm{TH}^{+}$survival (Doi et al., 2014). Alternatively, cultures were sorted late in the vm differentiation yet were not grafted (Xia et al., 2017).

With the rapid advancement of hPSC-derived DA progenitor grafts toward the clinic, cell-sorting studies have increasingly focused on the identification of cell surface proteins, to avoid reporter cell lines. A key challenge here has been the poor translation of findings from rodent to human, with several studies using rodent tissue to identify clinically applicable cell surface antigens on DA progenitor populations, yet translation of these approaches showed limited success as antibodies failed to show the same restrictive isolation or immunoreactivity in human cells (Bye et al., 2015; Gennet et al., 2016; Samata et al., 2016; and our unpublished observations). The narrowing attention to cell surface markers for selection has to some degree distracted from the primary goals of demonstrating the full potential of vmDA progenitor enrichment, and the safety goals.

Focused on these tasks of safety, predictability, and reproducibility, here we present the first evidence of using a fluorescent transgene to isolate and engraft hPSC-derived vm progenitors with superior outcomes to cell surface-based purification strategies. While we do not claim LMX1A as the optimal target gene (recognizing expression within other nondopaminergic vm progenitors), this work provides the important impetus for sorting using a human targeted gene. Moving forward, the recent work by Kirkeby et al. (2017) may provide new insight into additional and/or superior sorting candidates, having recently identified genes (including ETV5, EN1, SPRY1, WNT1, and CNPY1) expressed during vm differentiation that are predictive of positive DA graft outcomes. Interestingly, several genes commonly used to monitor DA differentiation (which have been used in sorting studies, e.g., FOXA2 and CORIN), have shown poor correlates with graft outcomes (Kirkeby et al., 2017). While our selected transgene, LMX1A, was shown to correlate positively with DA density in grafts (Kirkeby et al., 2017), the emphasis remains that additional markers are also likely needed. At this point, the field is now left to ponder the risk-to-benefit ratio of taking GFP cell lines into the clinic, noting that GFP transgenes have previously been used in tracing and targeted gene therapy clinical trials (www.clinicaltrials.gov) or the necessity of identifying a cell surface target.

In conclusion, improved preclinical sorting strategies may enable the identification of novel protein, epigenetic, or transcriptomic identifiers that underpin positive DA transplantation outcomes and therefore inform further clinically relevant interventions.

\section{References}

Aguila JC, Blak A, van Arensbergen J, Sousa A, Vázquez N, Aduriz A, Gayosso M, Lopez Mato MP, Lopez de Maturana R, Hedlund E, Sonntag KC, Sanchez-Pernaute R (2014) Selection based on FOXA2 expression is not sufficient to enrich for dopamine neurons from human pluripotent stem cells. Stem Cells Transl Med 3:1032-1042.

Andersson E, Tryggvason U, Deng Q, Friling S, Alekseenko Z, Robert B, Perlmann T, Ericson J (2006) Identification of intrinsic determinants of midbrain dopamine neurons. Cell 124:393-405.

Barker RA, Barrett J, Mason SL, Björklund A (2013) Fetal dopaminergic transplantation trials and the future of neural grafting in Parkinson's disease. Lancet Neurol 12:84-91.

Barker RA, Parmar M, Studer L, Takahashi J (2017) Human trials of stem cell-derived dopamine neurons for Parkinson's disease: dawn of a new era. Cell Stem Cell 21:569-573.

Bye CR, Thompson LH, Parish CL (2012) Birth dating of midbrain dopamine neurons identifies A9 enriched tissue for transplantation into parkinsonian mice. Exp Neurol 236:58-68.

Bye CR, Jönsson ME, Björklund A, Parish CL, Thompson LH (2015) Transcriptome analysis reveals transmembrane targets on transplantable midbrain dopamine progenitors. Proc Natl Acad Sci U S A 112:E1946-E1955.

Carlsson T, Carta M, Winkler C, Björklund A, Kirik D (2007) Serotonin neuron transplants exacerbate L-DOPA-induced dyskinesias in a rat model of Parkinson's disease. J Neurosci 27:8011-8022.

Denham M, Bye C, Leung J, Conley BJ, Thompson LH, Dottori M (2012) Glycogen synthase kinase 3beta and activin/nodal inhibition in human embryonic stem cells induces a pre-neuroepithelial state that is required for specification to a floor plate cell lineage. Stem Cells 30:2400-2411.

Doi D, Samata B, Katsukawa M, Kikuchi T, Morizane A, Ono Y, Sekiguchi K, Nakagawa M, Parmar M, Takahashi J (2014) Isolation of human induced pluripotent stem cell-derived dopaminergic progenitors by cell sorting for successful transplantation. Stem Cell Reports 2:337-350.

Freeman TB, Sanberg PR, Nauert GM, Boss BD, Spector D, Olanow CW, Kordower JH (1995) The influence of donor age on the survival of solid and suspension intraparenchymal human embryonic nigral grafts. Cell Transplant 4:141-154.

Fukuda H, Takahashi J, Watanabe K, Hayashi H, Morizane A, Koyanagi M, Sasai Y, Hashimoto N (2006) Fluorescence-activated cell sorting-based purification of embryonic stem cell-derived neural precursors averts tumor formation after transplantation. Stem Cells 24:763-771.

Ganat YM, Calder EL, Kriks S, Nelander J, Tu EY, Jia F, Battista D, Harrison N, Parmar M, Tomishima MJ, Rutishauser U, Studer L (2012) Identification of embryonic stem cell-derived midbrain dopaminergic neurons for engraftment. J Clin Invest 122:2928-2939.

García J, Carlsson T, Döbrössy M, Nikkhah G, Winkler C (2011) Impact of 
dopamine to serotonin cell ratio in transplants on behavioral recovery and L-DOPA-induced dyskinesia. Neurobiol Dis 43:576-587.

Gennet N, Tamburini C, Nan X, Li M (2016) FolR1: a novel cell surface marker for isolating midbrain dopamine neural progenitors and nascent dopamine neurons. Sci Rep 6:32488.

Grealish S, Diguet E, Kirkeby A, Mattsson B, Heuer A, Bramoulle Y, Van Camp N, Perrier AL, Hantraye P, Björklund A, Björklund A, Parmar M (2014) Human ESC-derived dopamine neurons show similar preclinical efficacy and potency to fetal neurons when grafted in a rat model of Parkinson's disease. Cell Stem Cell 15:653-665.

Hedlund E, Pruszak J, Lardaro T, Ludwig W, Viñuela A, Kim KS, Isacson O (2008) Embryonic stem cell-derived Pitx3-enhanced green fluorescent protein midbrain dopamine neurons survive enrichment by fluorescenceactivated cell sorting and function in an animal model of Parkinson's disease. Stem Cells 26:1526-1536.

Holmqvist S, Brouwer M, Djelloul M, Diaz AG, Devine MJ, Hammarberg A, Fog K, Kunath T, Roybon L (2015) Generation of human pluripotent stem cell reporter lines for the isolation of and reporting on astrocytes generated from ventral midbrain and ventral spinal cord neural progenitors. Stem Cell Res 15:203-220.

Jönsson ME, Ono Y, Björklund A, Thompson LH (2009) Identification of transplantable dopamine neuron precursors at different stages of midbrain neurogenesis. Exp Neurol 219:341-354.

Kauhausen J, Thompson LH, Parish CL (2013) Cell intrinsic and extrinsic factors contribute to enhance neural circuit reconstruction following transplantation in parkinsonian mice. J Physiol 591:77-91.

Kee N, Volakakis N, Kirkeby A, Dahl L, Storvall H, Nolbrant S, Lahti L, Björklund AK, Gillberg L, Joodmardi E, Sandberg R, Parmar M, Perlmann T (2017) Single-cell analysis reveals a close relationship between differentiating dopamine and subthalamic nucleus neuronal lineages. Cell Stem Cell 20:29-40.

Kirkeby A, Grealish S, Wolf DA, Nelander J, Wood J, Lundblad M, Lindvall O, Parmar M (2012) Generation of regionally specified neural progenitors and functional neurons from human embryonic stem cells under defined conditions. Cell Rep 1:703-714.

Kirkeby A, Nolbrant S, Tiklova K, Heuer A, Kee N, Cardoso T, Ottosson DR, Lelos MJ, Rifes P, Dunnett SB, Grealish S, Perlmann T, Parmar M (2017) Predictive markers guide differentiation to improve graft outcome in clinical translation of hESC-based therapy for Parkinson's disease. Cell Stem Cell 20:135-148.

Kriks S, Shim JW, Piao J, Ganat YM, Wakeman DR, Xie Z, Carrillo-Reid L, Auyeung G, Antonacci C, Buch A, Yang L, Beal MF, Surmeier DJ, Kordower JH, Tabar V, Studer L (2011) Dopamine neurons derived from human ES cells efficiently engraft in animal models of Parkinson's disease. Nature 480:547-551.

Lehnen D, Barral S, Cardoso T, Grealish S, Heuer A, Smiyakin A, Kirkeby A, Kollet J, Cremer H, Parmar M, Bosio A, Knöbel S (2017) IAP-based cell sorting results in homogeneous transplantable dopaminergic precursor cells derived from human pluripotent stem cells. Stem Cell Rep 9:1207-1220.

Mishima Y, Lindgren AG, Chizhikov VV, Johnson RL, Millen KJ (2009) Overlapping function of Lmxla and Lmxlb in anterior hindbrain roof plate formation and cerebellar growth. J Neurosci 29:11377-11384.
Nefzger CM, Su CT, Fabb SA, Hartley BJ, Beh SJ, Zeng WR, Haynes JM, Pouton CW (2012) Lmxla allows context-specific isolation of progenitors of GABAergic or dopaminergic neurons during neural differentiation of embryonic stem cells. Stem Cells 30:1349-1361.

Niclis JC, Gantner CW, Alsanie WF, McDougall SJ, Bye CR, Elefanty AG, Stanley EG, Haynes JM, Pouton CW, Thompson LH, Parish CL (2017a) Efficiently specified ventral midbrain dopamine neurons from human pluripotent stem cells under xeno-free conditions restore motor deficits in parkinsonian rodents. Stem Cells Transl Med 6:937-948.

Niclis JC, Gantner CW, Hunt CP, Kauhausen JA, Durnall JC, Haynes JM, Pouton CW, Parish CL, Thompson LH (2017b) A PITX3-EGFP reporter line reveals connectivity of dopamine and non-dopamine neuronal subtypes in grafts generated from human embryonic stem cells. Stem Cell Rep 9:868-882.

Politis M, Oertel WH, Wu K, Quinn NP, Pogarell O, Brooks DJ, Björklund A, Lindvall O, Piccini P (2011) Graft-induced dyskinesias in Parkinson's disease: high striatal serotonin/dopamine transporter ratio. Mov Disord 26:1997-2003.

Pruszak J, Sonntag KC, Aung MH, Sanchez-Pernaute R, Isacson O (2007) Markers and methods for cell sorting of human embryonic stem cellderived neural cell populations. Stem Cells 25:2257-2268.

Reyes S, Fu Y, Double K, Thompson L, Kirik D, Paxinos G, Halliday GM (2012) GIRK2 expression in dopamine neurons of the substantia nigra and ventral tegmental area. J Comp Neurol 520:2591-2607.

Samata B, Doi D, Nishimura K, Kikuchi T, Watanabe A, Sakamoto Y, Kakuta J, Ono Y, Takahashi J (2016) Purification of functional human ES and iPSC-derived midbrain dopaminergic progenitors using LRTM1. Nat Commun 7:13097.

Smidt MP, Smits SM, Burbach JP (2004) Homeobox gene Pitx3 and its role in the development of dopamine neurons of the substantia nigra. Cell Tissue Res 318:35-43.

Somaa FA, Wang TY, Niclis JC, Bruggeman KF, Kauhausen JA, Guo H, McDougall S, Williams RJ, Nisbet DR, Thompson LH, Parish CL (2017) Peptide-based scaffolds support human cortical progenitor graft integration to reduce atrophy and promote functional repair in a model of stroke. Cell Rep 20:1964-1977.

Thompson LH, Andersson E, Jensen JB, Barraud P, Guillemot F, Parmar M, Björklund A (2006) Neurogenin2 identifies a transplantable dopamine neuron precursor in the developing ventral mesencephalon. Exp Neurol 198:183-198.

Xia N, Fang F, Zhang P, Cui J, Tep-Cullison C, Hamerley T, Lee HJ, Palmer T, Bothner B, Lee JH, Pera RR (2017) A Knockin Reporter Allows Purification and Characterization of mDA Neurons from Heterogeneous Populations. Cell Rep 18:2533-2546.

Torres EM, Monville C, Gates MA, Bagga V, Dunnett SB (2007) Improved survival of young donor age dopamine grafts in a rat model of Parkinson's disease. Neuroscience 146:1606-1617.

Yan CH, Levesque M, Claxton S, Johnson RL, Ang SL (2011) Lmxla and $\operatorname{lmx} 1 \mathrm{~b}$ function cooperatively to regulate proliferation, specification, and differentiation of midbrain dopaminergic progenitors. J Neurosci 31: 12413-12425. 